\title{
Fuzzy Inference System for Physical Activity Recognition Based on General Features and PCA
}

\author{
Tongbi Kang, Lian Ying, Jiankang Wu, Yi Sun \\ University of Chinese Academy of Sciences, \\ Beijing, China. \\ E-mail: kangtongbi11@mail.ucas.ac.cn, \{jilianying, \\ wujiankang, sunyi\}@ucas.ac.cn
}

\author{
Xiaoli Meng \\ National University of Singapore, \\ Singapore. \\ E-mail: xiaoli.meng09@gmail.com
}

\begin{abstract}
This paper presents an approach of applying principal component analysis (PCA) and fuzzy inference system (FIS) to recognition of activity of daily life (ADL). To overcome the non-intuitiveness of single acceleration signal and difficulties of feature selection manually, 32 common features are computed and PCA is used for feature reduction. The membership functions are obtained from training data, and fuzzy rules are in same form for all classes. Thus, the FIS is not dependent on expert knowledge of physical activity. Thus, this system is extendable on new types of activity, new features or new locations. This system is designed for a real-time sensorbased monitoring system to recognize 6 types of daily physical activities. Sitting, standing, walking, going upstairs, going downstairs and running are classified with a precision of $99.78 \%, 90.78 \%, 91.89 \%, 89.72 \%, 91.28 \%$ and $100 \%$ for each type.
\end{abstract}

Keywords-physical activity recognition; principal component analysis; fuzzy inference system

\section{INTRODUCTION}

Physical activity monitoring can be used for healthcare services for patients and elder people [1]. Practical applications include falling detection, energy expenditure monitoring and physical therapy management. As for this ubiquitous scenario, MEMS-based inertial sensors such as accelerometers, gyroscopes and magnetometers are widely accepted because they are small in size and consume less power. Using signals from those sensors for physical activity recognition is a challenge because they are usually not enough in number to obtain all movement information of the whole body and the signals from them are not intuitive as well.

Various methods have been proposed for physical activity recognition, which can be found in two reviews of [1], [3]. Karantonis, Mathie, et al. used one waist-worn accelerometer and a real-time threshold-based classification algorithm to recognize daily activities. They also used waistworn sensor to recognize activity patterns for the elder. Bao et al. used five bi-axis accelerometers to recognize activities of daily life (ADL), and they tried decision table, instancebased learning (IBL or nearest neighbor), C4.5 decision tree, and naive Bayes classifiers. Amft et al. used four arm-worn sensors and HMM to monitor the activity of eating and drinking. Sugimoto et al. used one wristwatch-type motion sensor and foot pressure sensing shoes to recognize the motion of thigh by a self-define threshold-based classification algorithm.

The idea of Fuzzy logic is identical with intrinsically obscureness of the definition of human postures and activities. As mentioned before, sensors limited in number can only represent part of physical activity information. In this case, fuzzy concept can also explain the uncertain zones between activity definitions. Lee, Mase, et al. used fuzzy classifier to differentiate human gaits with an accuracy of $90 \%$. Here Gyro-scope signal was used to define membership functions and "AND" operation was used to define a set of rules [4]. Salarian et al. used the Mamdani fuzzy inference method. This classifier was developed using membership functions constructed from knowledge of activity states before and after the period of interest [2]. Classification accuracies obtained by this approach were shown to be better than those obtained using simple threshold rules [2]. Boissy et al. used Mamdani fuzzy inference to identify falls. A tri-axial accelerometer was used as input to a fuzzy classifier. Using a large dataset of fall and non-fall events from 10 subjects, they were able to demonstrate average fall detection accuracies ranging between 86 and 93\%, depending on sensor location [5].

One of the challenges using fuzzy system to classify human activity is that raw acceleration signal is not intuitive and the intuitive measurements such as speed or displacement cannot be obtained by integrating acceleration signal because of sensor drift. As a result, it is not easy to find one or several features with well-defined fuzzy membership functions to classify physical activities. Therefore, a wide type of features must be involved and feature selection or reduction method must be used to build fuzzy system. In this paper, 32 features are introduced and principal component analysis (PCA) is used for reducing the large feature set. Then using training data, membership functions are obtained without any manual intervention, because the physical explanations of the principal components are obscure. Thus, the fuzzy inference system (FIS) is not dependent on expert knowledge of physical activity. With this approach, a Mamdani FIS is designed for recognizing as many as 6 main daily activities using a triaxis accelerometer worn on thigh, and overall accuracy of $94 \%$ is achieved. 


\section{DATA Collection AND PREPROCESSING}

The assumed application scenario is monitoring activities in real-time way. Here, real-time means when classifying, only short term data until now is used. This system shown in Figure 1 consists of wearable sensor node, communication gateway, a mobile phone, on each subject and a remote server located in monitoring center.

The sensor node contains a tri-axis digital accelerometer, ADXL345 from Analog Devices Inc. The sampling rate is $50 \mathrm{~Hz}$, the sampling resolution is 8 bits, and measurement range is set to $\pm 2 \mathrm{~g}$. The sensor node is worn on subject's thigh.

A micro-controller and a Bluetooth module are also included on the sensor node for control and data transmission to the mobile phone. Using WI-FI or cellular network, the mobile phone transmits the data to the central server, where the data processing and classification are conducted.

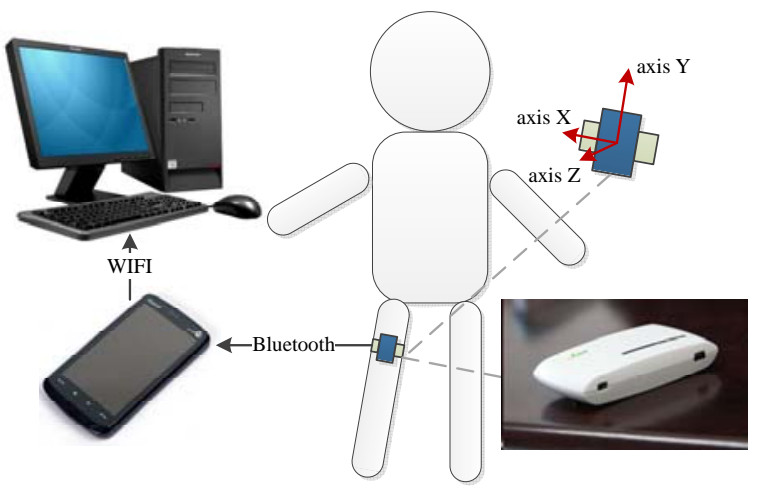

Figure 1. Data collection system and axis definition

To determine the activity type in real-time way, the acceleration signal samples are segmented into window series and only newest 50 acceleration signal samples are used for classification for each window. ${ }^{1}$ The number 50 means time window length of one second, which is enough to include one typical movement cycle such as one walking step.

The raw data contains noises and outliers caused by sensor noises, environmental interference or electronic disturbance. Denoising is necessary for improving the quality of the signal. A 50-order high-pass Bartlett-Hanning window FIR filter is designed using MATLAB, and cutoff frequency is set to $20 \mathrm{~Hz}$.

\section{FEATURE EXTRACTION AND REDUCTION}

The activities and postures to be classified include sitting, standing, walking, going upstairs, going downstairs and running. As mentioned in the first section, the challenge of this classification is that human movements are complex, their difference in the acceleration signals are not intuitive

\footnotetext{
${ }^{1}$ For most features, it is the case. Except for frequency-domain features calculating FFT, it is 100 acceleration samples, the first 50 of which is overlapped with the previous window and similarly for wavelet analysis, it is 200 in this paper with the first 150 overlapped.
}

and not easy to identify. Therefore, in this study, 32 features will be extracted, which are commonly used in this domain. The large number of features will require large sample size for training. Furthermore, they are usually correlated with each other. For example, both SMA and PPA (introduced later in this section) reflect the activity intensity. In result, feature space reduction is necessary, and PCA is utilized for this aim.

\section{A. Feature extraction}

The features computed can be categorized into heuristic features, time-domain features, frequency-domain features and time-frequency-domain features. The first type of features is highly related to the physical principles of the human activity, and the last three types of features are for general signal analysis [1].

1) Heuristic features. The heuristic features selected are Signal Magnitude Area (SMA) in (1), Peak-to-peak Amplitude of three axes (PPAx, PPAy, PPAz) [1], correlation between axes (Corr_xy, Corr_yz, Corr_xz).

SMA stands for the area under the high pass filtered acceleration curve. In (1), $a_{x} a_{y} a_{z}$ are acceleration of axes $x y z$.

$$
S M A=\frac{1}{t} \int_{0}^{t}\left(\left|a_{x}(\tau)\right|+\left|a_{y}(\tau)\right|+\left|a_{z}(\tau)\right|\right) \mathrm{d} \tau
$$

Many previous researches proved that SMA is closely related to motion energy and some of the application research use SMA as a close estimation of motion energy [6]. This indicates that SMA can be used to divide different energy levels of activity.

Peak-to-peak amplitude is the range between neighbor peaks of the signal, which represents the changing amplitude of the signal, namely activity intensity. It is similar with SMA and can be used as supplement for representing signal energy.

Correlation between axes can be used for presenting the motion pattern. For example, when comparing walking to running, the correlation between front-back and left-right axis is larger in walking than in running. This can be explained as people tend to act in more relaxed paces when walking and this movement has better coordination than running.

2) Time-domain features.Time-domain features selected are mean, standard deviation, skewness and kurtosis, which are calculated for each window. Mean is mainly used for detecting postures. Variation discerns static postures and kinetic activities. Skewness is the degree of asymmetry in the distribution of acceleration data, and kurtosis is a measure reflecting how much a distribution is peaked at the center of a distribution [6]. The last two features are particularly useful for recognizing implicit motion pattern differences between walk, going upstairs and downstairs.

3) Frequency-domain features. Frequency domain features selected are dominant frequency, frequency-domain entropy [1] and spectral energy in (2). Fast Fourier 
transform (FFT) is firstly operated on windows. For motions have periodicity like walking, running, going upstairs and downstairs, frequency components are distributed in apparent patterns, usually concentrated on several frequency bands. Features mentioned above are used to describe the characteristic of these frequency bands. Dominant frequency, $f_{\text {Dominant }}$, stands for the largest frequency of the signal, which is used to differentiate different activities with periodicity like the pace of walking, going upstairs/downstairs, running, cycling and so forth.

This paper assumes $A_{v}(f)$ is the corresponding Fourier coefficients for signal $a_{v}$ ( $\mathrm{v}$ is one of xyz axis).

As for frequency-domain entropy, it is the normalized information entropy of the FFT coefficient magnitude of the signal. Firstly, the spectrum is normalized in (2),

$$
a^{\text {norm }}(f)=A(f) / \int A(f) \mathrm{d} f
$$

and then use the result for entropy, $H$, calculation in (3).

$$
H\left(a^{\mathrm{norm}}\right)=-\int a^{\mathrm{norm}}(f) \cdot \log _{2} a^{\text {norm }}(f) \mathrm{d} f
$$

This feature can interpret the different width of frequency band in the activities mentioned above.

Spectral energy, $E_{\text {Spectral }}$, defined in (4) is the sum of squared FFT coefficients which is used for variation of the frequency spectrum.

$$
E_{\text {Spectral }}=\int\left|A_{v}(f)\right|^{2} \mathrm{~d} f
$$

For example, spectral energy for running is higher than walking as it includes more irregular quivering of the muscles on leg.

4) Time-frequency-domain features. Wavelet analysis is famous for its ability for investigating both the time and frequency characteristics, comparing to Fourier analysis. Here wavelet Daubechies 6 (db6) is selected for discrete wavelet transform and the signal is decomposed up to level 5. The approximation of wavelet coefficients on level 4 (a4) is discernible for going upstairs and downstairs. From Figure 2, it is obvious that the coefficients magnitude and the signal energy are all in different levels between going upstairs and downstairs.

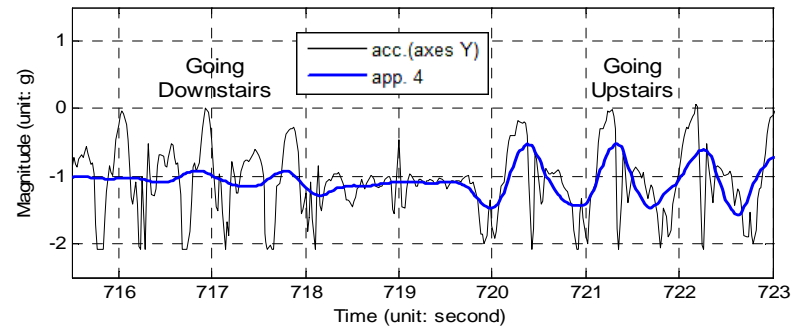

Figure 2. Signal and approximation at level 4 after db6 wavelet analysis
After calculating the SMA of the a4 in each window, the result can be named as $\mathrm{SMA}_{\mathrm{WT}}$ defined in (5) and presented in Figure 3, which is one time-frequency feature.

$$
S M A_{\mathrm{WT}}=\sum_{v \in x, y, z} \frac{1}{t}\left(\int_{0}^{t}\left|\operatorname{app}_{v}^{4}(\tau)\right| \mathrm{d} \tau\right)
$$

In this equation, $\left|\operatorname{app}_{v}^{4}(\tau)\right|$ stands for a4 mentioned above.

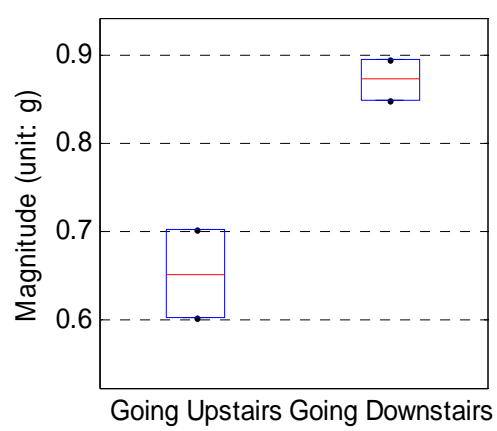

Figure 3. Feature extracted from wavelet analysis, $\mathrm{SMA}_{\mathrm{WT}}$

\section{B. Dimensionality reduction using PCA}

Principal component analysis is a quantitatively rigorous method for feature space reduction. The method generates a new set of variables, called principal components [7]. The principal components as a whole form an orthogonal basis for the space of the data. The components generated by PCA are perpendicular to each other. The variance of these components decreases in the order of generation. Thus, components with bigger variance can be selected to represent data with trivial loss, which can be used for feature reduction. The procedure of using PCA to reduce the dimension of the whole data set is as follows.

1) Normalizing pre-processed feature data. In the provided data set, the features are in different units and the variance of the different columns is substantial. Therefore, it is appropriate normalizing the data by dividing each column by its standard deviation.

2) Principal Component Analysis. PCA computation generates a new set of variables which are linear combination of original features. The computation can be achieved by singular value decomposition.

\section{Feature reduction}

After PCA, the coefficients are recorded for new feature generation. Here 32 principal components are generated by multiplying the original feature set to the coefficients. In order to select the most helpful components for the classification, the percent of the total variability explained by each principal component is calculated. The first eight components represent nearly $95 \%$ of the complete feature space. As a result, the first eight components are used for further classification, the inputs of the FIS, considering both preservation of the maximum feature set information and easiness to construct the FIS. 


\section{FIS CONSTRUCTION AND CLASSIFICATION}

Fuzzy inference system originates from fuzzy set theory and fuzzy mathematics. In FIS, the inputs that are not fuzzy are firstly fuzzified according to member function, which are defined by the designer and problem dependent. Then the fuzzy inputs are mapped into outputs according to a set of fuzzy rules and logic. Again, the fuzzy rules and logic are defined by the designer and problem dependent. The mapped outputs are then aggregated into one fuzzy output, which can be used for inference or classification decision.

For our physical activity recognition problem, features extracted from sensor signals and selected via PCA are the inputs, with the fuzzy outputs representing each type of activity. Based on the previous description, the construction of FIS in this paper is demonstrated in Figure 4 and implemented by a definition of membership function, IFTHEN rules and aggregation as follows.

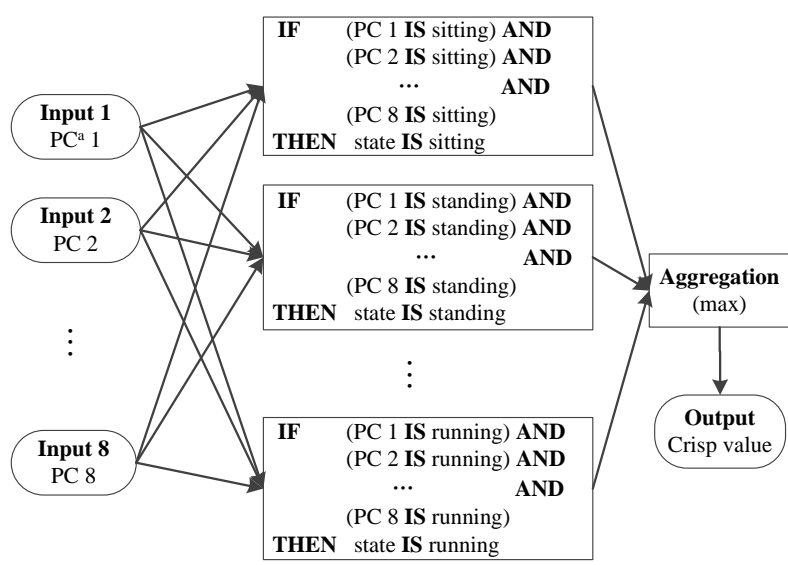

${ }^{\text {a }}$ Principal components after PCA

Figure 4. Construction of FIS

\section{A. Membership function}

In contrast to classical set theory in which a data point's membership is either in or out, by allowing the membership function to range between 0 and 1 , fuzzy set theory permits partial membership in multiple sets.

Definition of the membership function is based on the distribution of training inputs on different types of activity. Distributions of inputs, namely the principal components, are close to Gauss distribution, which is taken as our membership functions. The parameters of Gaussian distribution can be estimated by the training inputs.

\section{B. IF-THEN rules}

Using the membership function, every input will be assigned a membership number. Then Fuzzy logic and rules are used to map these numbers to outputs. There can be more than one rule. In this application, 6 rules are defined, each of which are corresponding to one activity type. For each rule, 8 inputs, corresponding to 8 features, are used, and 1 output is mapped.
Fuzzy IF-THEN rules are expressions of the form IF A THEN B, where A and B are called the antecedent and the consequent, respectively. These rules are generated by applying fuzzy operators to the inputs and outputs.

For this application, the rules have the form shown in Figure 4. For the rule, "PC 1 IS running", "IS" means membership, namely, how much PC 1 value belongs to running PC 1 set. The expression is a number ranging from 0 to 1 . In the same way, all the PCs will be evaluated to generate membership number. Those numbers are then fed into fuzzy operations, namely, the "AND" operation in this application. In Fuzzy logic, we can use "min” for AND operator. Using “AND” operations, 8 membership numbers are combined into one antecedent number.

At last, the IF-THEN rule is applied, which uses the antecedent number to imply the consequent number. Implication is the process of shaping the fuzzy set in the consequent based on the results of the antecedent in a FIS [8]. Again, “min” is used for implication of each rule.

\section{Aggregation}

The final step of FIS is aggregation. It combines the 8 outputs of fuzzy rules and forms a single fuzzy set, which is later used for a defuzzification method to determine the final activity class. Here "max" is used for aggregation. And defuzzification method is "centroid" which returns the center of area under the curve [8].

A usage of the FIS can be shown by applying an example input to it. The sample [22.5 -9.81 -7.38 -5.5 3.02 3.94 2.28 1.96] is applied to this FIS, and firstly inputs to the membership function (fuzzification), applying the fuzzy operators to the different parts of the antecedents, implication, aggregation, and finally gives a crisp output, 0.53 (defuzzification). (Fig. 5)

From training group, the crisp output can be gathered for each type of activity. And by analyzing the distribution of the crisp output for each type, the type of the activity can be classified based on Table I. For the sample given above, it is classified as sitting state.

TABLE I. RANGE OF CRISP OUTPUT FOR EACH TYPE

\begin{tabular}{|c|c|}
\hline Type of activity & $\begin{array}{c}\text { Range of crisp } \\
\text { output }\end{array}$ \\
\hline Sitting & $0 \sim 0.54$ \\
\hline Standing & $0.56 \sim 0.70$ \\
\hline Walking & $0.54 \sim 0.55$ \\
\hline Going upstairs & $0.55 \sim 0.56$ \\
\hline Going downstairs & $0.70 \sim 0.85$ \\
\hline Running & $0.85 \sim 1$ \\
\hline
\end{tabular}

\section{EXPERIMENTS AND RESULTS}

Data of 6 types daily physical activities are collected with each type lasting for 15 minutes, which means each type is repeated many times. The 6 types are sitting, standing, walking, going upstairs, going downstairs and running. Data length for each type is more than 900 seconds. All the activities that accomplished by recruited volunteers were 
performed in the real-life environment without following certain instructions from researcher.

Cross validation is used to evaluate the performance of the FIS. 50\% of the data are used for training to get the fuzzy membership functions and crisp function, and another 50\% of the data are used for testing. The result is compared with the other FIS without using PCA in [8] tested on the same dataset, which is shown in Table II.

TABLE II. SENSITIVITY, PRECISION AND FALSE POSITIVE RATE IN EACH ACTIVITY

\begin{tabular}{|c|c|c|c|c|c|c|}
\hline \multirow{2}{*}{$\begin{array}{c}\text { Type of } \\
\text { activity }\end{array}$} & \multicolumn{2}{|c|}{ Sensitivity (\%) } & \multicolumn{2}{c|}{ Precision (\%) } & \multicolumn{2}{c|}{$\begin{array}{c}\text { False positive } \\
\text { rate (\%) }\end{array}$} \\
\cline { 2 - 7 } & $\begin{array}{c}\text { PCA- } \\
\text { FIS }\end{array}$ & $\begin{array}{c}\text { Simple } \\
\text { FIS }\end{array}$ & $\begin{array}{c}\text { PCA- } \\
\text { FIS }\end{array}$ & $\begin{array}{c}\text { Simple } \\
\text { FIS }\end{array}$ & $\begin{array}{c}\text { PCA- } \\
\text { FIS }\end{array}$ & $\begin{array}{c}\text { Simple } \\
\text { FIS }\end{array}$ \\
\hline Sitting & $\mathbf{9 9 . 8}$ & 83.2 & $\mathbf{9 9 . 8}$ & 83.2 & $\mathbf{0 . 2}$ & 16.8 \\
\hline Standing & $\mathbf{9 0 . 8}$ & 83.1 & $\mathbf{9 0 . 8}$ & 83.1 & $\mathbf{9 . 2}$ & 16.9 \\
\hline Walking & $\mathbf{9 1 . 9}$ & 87.9 & $\mathbf{9 1 . 9}$ & 87.9 & $\mathbf{8 . 1}$ & 12.1 \\
\hline Go upstairs & $\mathbf{8 9 . 7}$ & 88.6 & $\mathbf{8 9 . 7}$ & 88.6 & $\mathbf{1 0 . 3}$ & 11.4 \\
\hline Go downstairs & $\mathbf{9 1 . 3}$ & 84.1 & $\mathbf{9 1 . 3}$ & 84.1 & $\mathbf{8 . 7}$ & 15.9 \\
\hline Running & $\mathbf{1 0 0 . 0}$ & 99.3 & $\mathbf{1 0 0 . 0}$ & 99.3 & $\mathbf{0 . 0}$ & 0.7 \\
\hline
\end{tabular}

Our FIS using more features and feature reduction perform better in activity recognition accuracy, which prove $\mathrm{s}$ that it is necessary to taken into more features while feature reduction can be used to reduce the feature size.

\section{CONCLUSIONS}

The FIS is suitable for physical activity classification because the intrinsic obscure nature of human activity definition and the lack of whole body movement information caused by one single sensor usage.

In this paper, classification using a tri-axis accelerometer with FIS is studied. Considering the non-intuitiveness of acceleration signal and to meet the need of finding proper features manually to build the FIS, 32 general features are introduced. To overcome the side effect of large feature size,
PCA is used for feature reduction. The principal components are used as inputs of the FIS. The performance is improved compared with FIS with only manually selected features.

Considering the usage of general features and automatic membership function generation of FIS, the system is automatic in sense of that no expert knowledge is required. Thus, the system is extendable on new types of activity, new features or new locations. This can be seen as another advantage of using general feature sets and feature reduction technique to build FIS for physical activity recognition.

\section{REFERENCES}

[1] S. J. Preece, J. Y. Goulermas, L. P. J. Kenney, Dave Howard, Kenneth Meijei and Obin Crompton, "Activity identification using bodymounted sensors-a review of classification techniques,”. Physiological Measurement, vol. 30, iss. 4, pp. R1-R33, 2009.

[2] A. Salarian, H. Russmann, F. J. G. Vingerhoets, P. R. Burkhard and K. Aminian, "Ambulatory monitoring of physical activities in patients with Parkinson's disease,” J. IEEE Trans. Biomed. Eng., vol. 54, iss. 12, pp. 2296-9, 2007.

[3] C. C. Yang and Y. L. Hsu, "A Review of Accelerometry - Based Wearable Motion Detectors for Physical Activity Monitoring," Sensors 2010, vol. 10, iss. 8, pp. 7772-7788, 2010.

[4] S. W. Lee and K. Mase, "Activity and location recognition using wearable sensors,” J. IEEE Perv. Comp., vol. 1, iss. 3, pp. 24-32, 2002.

[5] P. Boissy, S. Choquette, M. Hamel and N. Noury, "User-based motion sensing and fuzzy logic for automated fall detection in older adults,” J. Telemed J. E. Health., vol.13, iss. 6, pp. 683-693, 2007.

[6] A. G. Li, L. Y. Ji, S. F. Wang and J. K. Wu, "Physical Activity Classification Using a Single Triaxial Accelerometer Based on HMM,” IET International Conference on Wireless Sensor Network, Beijing, China. pp. 155-160. November 2010,

[7] J. E. Jackson, A User's Guide to Principal Components. John Wiley and Sons, 1991, pp. 592.

[8] M. Helmi and S. M. T. Almodarresi, "Human activity recognition using a fuzzy inference system,” 18th IEEE International Conference on Fuzzy Systems, vol. 1-3, pp. 1897-1902, 2009.
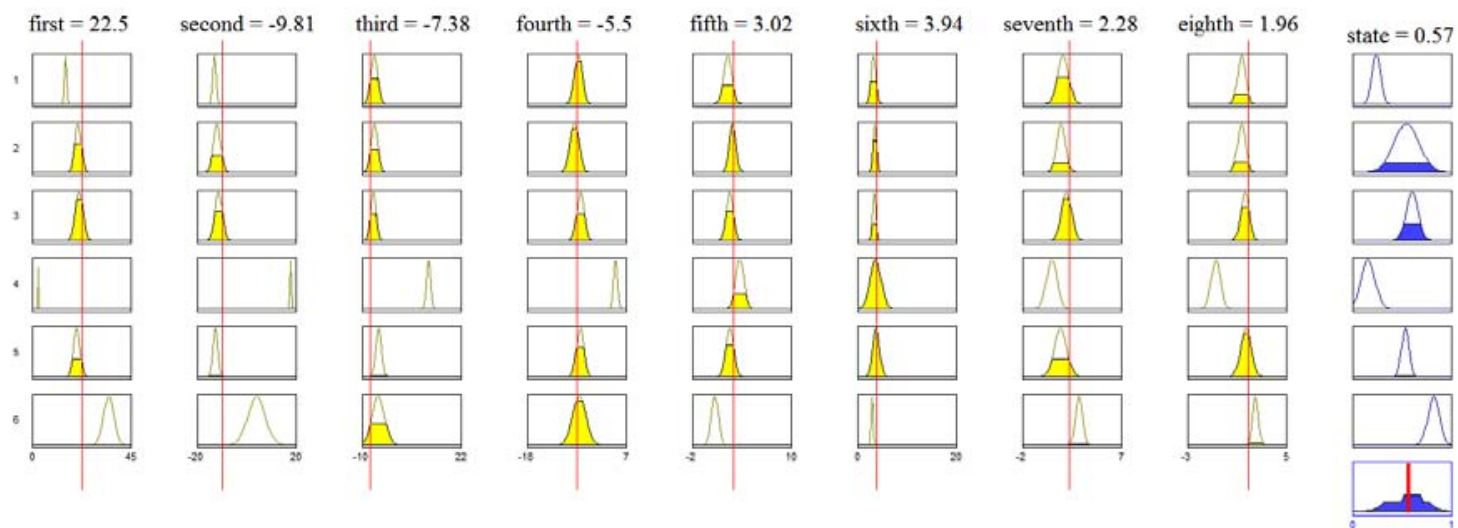

Figure 5. Fuzzification and defuzzification of input [22.5 -9.81 -7.38 -5.5 3.023 .942 .28 1.96] 